\title{
Le Laboratoire de physique de la matière condensée de Nice encourage et accompagne l'innovation
}

Le cliché est tenace : d'un côté des chercheurs académiques rétifs à l'idée de valoriser leurs résultats, de l'autre des industriels sans le moindre intérêt pour la recherche fondamentale. Et, au milieu, de jeunes chercheurs dont le travail de thèse n'aurait aucune chance de connaître une seconde vie au-delà des portes du laboratoire. Or, la preuve du contraire est donnée par le Laboratoire de physique de la matière condensée (LPMC), à Nice, où la fibre entrepreneuriale des doctorants est non seulement encouragée, mais également accompagnée et ce, éventuellement jusqu'à la création d'une entreprise innovante.

De quoi tirer le meilleur parti de l'alliance entre problématiques fondamentales et innovation industrielle.

\section{Une startup spécialisée dans la production de pigments naturels}

Le cas de Nicolas Volle, qui a soutenu sa thèse fin 2011, est à cet égard emblématique. Moins de deux ans plus tard, avec plusieurs prix en poche, dont le «Prix spécial 2012 » de la Fondation Norbert Segard ${ }^{(a)}$, le prix «Jeune créateur d'entreprise » 2012 délivré par la Fondation Unice, et le Prix de l'entreprise 2012 remis à Nice lors des Entreprenariales, le voilà président de PIGM'Azur. Cette jeune startup est spécialisée dans la production de pigments naturels hybrides organiquesinorganiques, qui ont la particularité d'être extrêmement stables, résistants et non toxiques. Ces propriétés intéressent le secteur industriel et, plus particulièrement, PIGM'Azur, qui ambitionne désormais de devenir incontournable pour des applications allant de la peinture aux cosmétiques, en passant par les plastiques et la papeterie.

L'histoire de Nicolas Volle est celle d'un jeune ingénieur décidé à mettre en valeur, avec toute la rigueur académique nécessaire, les propriétés et les performances d'un produit innovant, dans le but affiché de lui dessiner ensuite un avenir industriel. C'est ainsi qu'il a trouvé son bonheur au LPMC, dans un sujet proposé par Anne-Marie Chaze. Cette dernière, spécialiste de l'insertion de molécules organiques dans des cavités micrométriques, dirige une équipe qui a notamment mis au point un procédé permettant d'introduire à loisir des molécules organiques dans les tunnels et canaux qui criblent la surface d'argiles fibreuses, telle la sépiolite. Durant son doctorat, Nicolas Volle a pu mettre à profit ce procédé afin de synthétiser des pigments bleus à base d'argile et de colorants naturels, semblables à ceux obtenus empiriquement par les Mayas il y a 1600 ans et connus pour leur étonnante stabilité [1,2], de même qu'une large palette de couleurs.
La suite "résulte de la rencontre fructueuse entre un groupe de chercheurs ayant un goût pour la recherche appliquée et un doctorant animé par la volonté de créer une entreprise ", souligne Fabrice Mortessagne, directeur du LPMC. Nicolas Volle ajoute : "Je viens d'une famille d'entrepreneurs. Aller de la paillasse à la valorisation était donc pour moi une évidence ", mais dont la concrétisation tient pour une part importante à l'environnement rencontré au LPMC. "Nous avons apporté un soutien humain et logistique, précise Fabrice Mortessagne. Et je me suis assuré que Nicolas ait tous les bons contacts. " L'intéressé confirme : "J'ai pu, en particulier, étudier les opportunités avec le responsable valorisation du laboratoire. "Si bien qu'aujourd'hui, PIGM'Azur et le LPMC avancent main dans la main : la plupart des membres de l'équipe qui a entouré Nicolas Volle est partie prenante dans l'aventure. Quant à l'activité d'innovation de l'entreprise, "il est clair qu'elle s'appuiera très largement sur les compétences et l'infrastructure du LPMC », confirme F. Mortessagne.

\section{Mettre au point des composants végétaux pour peintures écologiques à séchage rapide}

Évidemment, le cas de Nicolas Volle fait figure d'exception. Mais comme l'indique F. Mortessagne, " le LPMC tisse des liens avec des industriels depuis au moins une quinzaine d'années, en particulier dans le cadre du co-financement de thèses. "Celle actuellement en cours de Matthieu Sonnati [3] est même financée à $100 \%$ par la société ECOAT. Et pour cause : après sa soutenance de thèse, le doctorant prendra la direction $\mathrm{R} \& \mathrm{D}$ de cette startup d'innovation primée par le ministère de la Recherche et par OSEO ! Comme il l'explique : "Début 2011, j'ai participé à la création de cette entreprise avec deux anciens collègues rencontrés chez un industriel de la chimie. Mais nous ne disposions pas de toute l'infrastructure de recherche nécessaire à l'exploration 


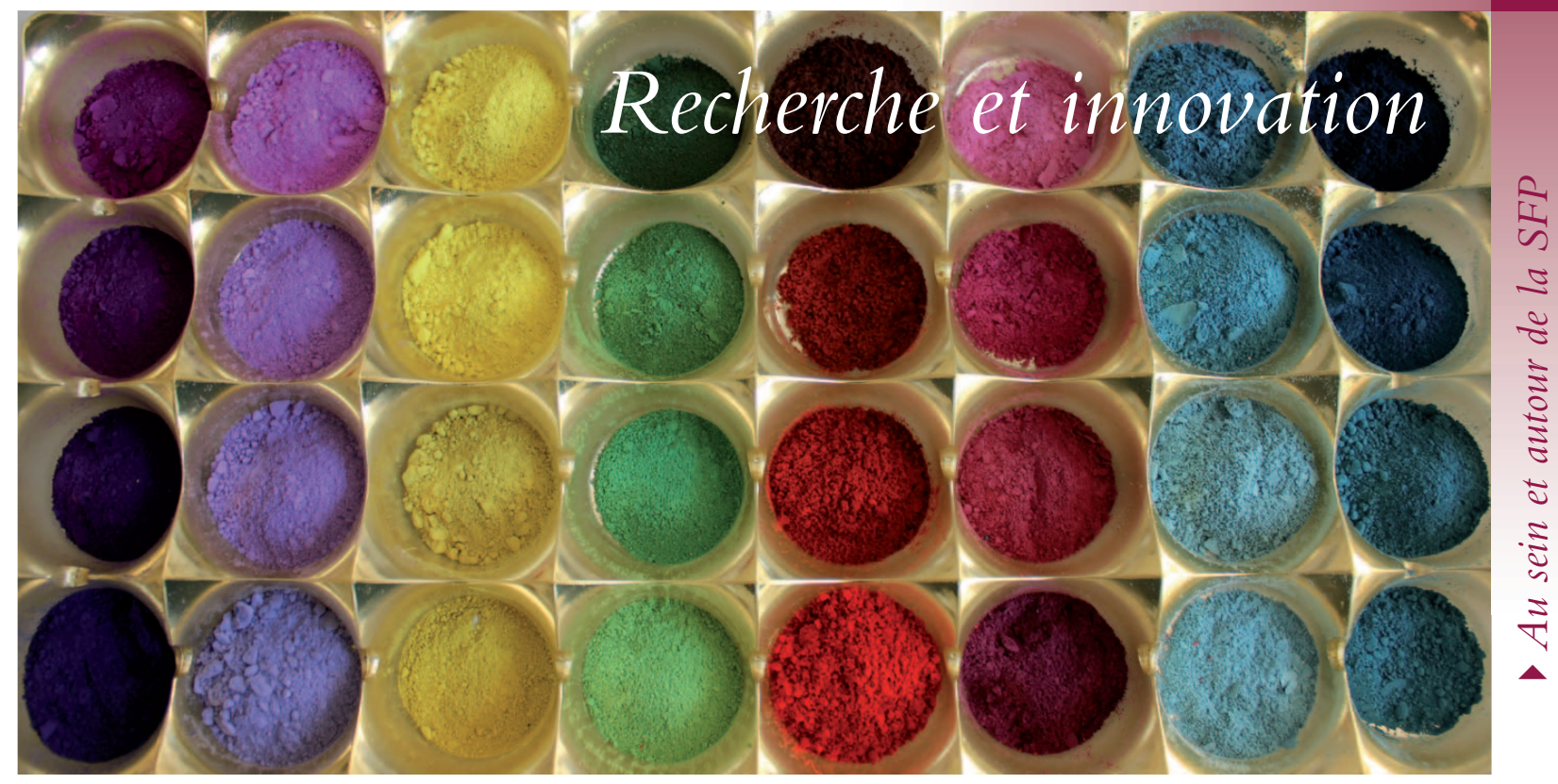

1. Pigments naturels produits par la société PIGM’Azur (c).

théorique et conceptuelle de nos innovations. C'est la raison pour laquelle je me suis rapproché du groupe de chimie des surfaces et interfaces de Frédéric Guittard, qui a été mon professeur lorsque j'étais étudiant en master. "

L'objectif : mettre au point un liant - le polymère qui constitue le film de peinture une fois le solvant évaporé - pour peinture, d'origine végétale, donc durable et non issu de dérivés pétroliferes. Secret industriel oblige, le jeune chercheur ne dira rien de sa recette. Mais comme il l'indique, "en revisitant complètement la structure moléculaire d'un film de peinture, nous $y$ sommes parvenus. " $\mathrm{Au}$ point que le procédé a conduit ECOAT en juillet 2012 au lancement sur le marché de deux premiers liants bio-sourcés à 95 et $99 \%$ ! À la suite de quoi Matthieu Sonnati, depuis lauréat de la première édition française du concours MIT Technology Review Innovateurs français de moins de 35 ans, est parvenu à mettre au point un procédé de séchage sans l'adjonction traditionnelle de sels métalliques, souvent toxiques. Ce projet est renforcé par une seconde thèse, débutée en 2012 dans le cadre d'une ANR conjointe d'ECOAT et du LPMC. "De part et d'autre, nous avons envie de conserver un lien fort", témoigne Fabrice Mortessagne, qui ajoute : "Les liens avec les entreprises sont importants pour notre laboratoire, même si le cour de notre métier est évidemment la recherche fondamentale. Cela permet de montrer à l'ensemble de nos doctorants que de multiples débouchés sont possibles, y compris dans l'industrie. "

\section{Les recherches en physique quantique ouvrent aussi des perspectives d'applications}

Fabrice Mortessagne en veut pour preuve le cas d'un de ses anciens étudiants ayant travaillé sur un problème très théorique de physique de la matière condensée, "mais qui a pourtant été embauché chez Thalès avant même sa soutenance de thèse ».
Ce cas de figure évoque un peu celui de Florian Kaiser, qui a récemment soutenu sa thèse au LPMC avant de poursuivre en postdoc dans le même laboratoire. La spécialité de ce jeune chercheur? C'est l'information quantique, une discipline dont les développements permettent à la fois de revisiter les fondements même de la mécanique quantique, mais également de proposer des systèmes, en particulier pour la cryptographie.

Dans ce domaine, Florian Kaiser, lauréat en 2013 du prix de thèse EPS Quantum Electronics and Optics Prize - Applied aspects de la Société européenne de physique, a mis au point trois nouvelles sources de photons intriqués, soit l'entité quantique de base permettant de mettre en œuvre des communications absolument inviolables $[4,5]$. Il est ainsi parvenu à créer une situation expérimentale où un photon ne se comporte pas, selon l'interprétation classique de la mécanique quantique, telle une onde ou une particule, mais tel un objet quantique irréductible. Une première ! Il n'empêche, " à l'issue de mon postdoc, je souhaite intégrer le monde industriel pour développer des lasers de puissance ", nous dit F. Kaiser. Signe indéniable que le LPMC, sans renier son statut de laboratoire de recherche fondamentale, sert de tremplin pour valoriser le travail de ses étudiants. I

Mathieu Grousson, journaliste.

(a) www.norbert-segard.org/menus/les-prix-norbert-segard/ les-finalistes-du-prix-2012/prix-special-2012-nicolas-volle
3- M.0. Sonnati et al., Green Chemistry, 15 (2013) 283

4• F. Kaiser et al. Laser Phys. Lett. 10 (2013) 045202

$5 \bullet$ F. Kaiser et al., Science 338 (2012) 637-640.

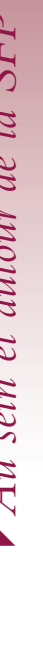

